\title{
Molecular Dynamics Simulations of Dynamic Force Microscopy: Applications to the $\operatorname{Si}(111)-7 \times 7$ Surface
}

\author{
A. Abdurixitt, A. Baratoff and E. Meyer \\ Department of Physics and Astronomy, University of Basel, Klingelbergstr. 82, 4056 Basel, Switzerland
}

(Accepted in Applied Surface Science)

\begin{abstract}
Molecular dynamics simulations have been performed to understand true atomic resolution, which has been observed on the $\operatorname{Si}(111)-7 \times 7$ surface by dynamic force microscopy in ultra high vacuum(UHV). Stable atomic-scale contrast is reproduced in simulations at constant mean height above a critical tip-sample separation when monitoring the interaction force between tip and sample. Missing or additional adatoms can be recognized in such scans, although they are less well resolved than native adatoms. The resonance frequency shift, as well as arbitrary scans, e.g. at constant force can be computed from a series of force-distance characteristics. By means of dynamic simulations we show how energy losses induced by interaction with an oscillating tip can be monitored and that they occur even in the non-contact range.
\end{abstract}

PACS: 07.79.Lh; 31.15.Qg; 34.20.Cf; 61.16.Ch; 68.35.Bs

Keywords: non-contact atomic force microscopy; molecular dynamics; energy transfer; silicon

\section{INTRODUCTION}

Atomic force microscopy (AFM) has become an important method in fundamental and applied surface science able to obtain local information about surface structure and interactions, especially on nonconducting samples. In this method the surface is probed by measuring the interaction with a sharp tip or changes produced by that interaction. Atomic resolution can be obtained if the tip can approach the surface in the attractive regime without making contact. In such non-contact experiments it is difficult to measure this interaction force accurately while avoiding cantilever jump to contact. However, because the resonance frequency of the cantilever changes locally if the tip starts to be attracted to specific surface sites, it became possible to image surface atoms by maintaining a constant frequency shift $\Delta f$ via feedback control of the cantilever perpendicular displacement [1,2]. This shift can be measured very accurately using frequency demodulation 3. Jump to contact is avoided by using a large cantilever force constant $k$ and a large tip oscillation amplitude $A$, such that the restoring force $k A$ considerably exceeds the attractive force at the distance of closest approach. Under this condition, the tip-sample interaction weakly perturbs the harmonic motion of the tip, so that $\Delta f$ can be calculated from the force-distance dependence at any particular site, as first pointed out by Giessibl [4]

Following the pioneering experiments [1,2], many investigators have obtained atomically-resolved images of the $\mathrm{Si}(111)-7 \times 7$ surface in ultra high vacuum (UHV) using large-amplitude dynamic force microscopy (DFM) under various feedback conditions 5 10. In particular Lüthi et al \&] recorded simultaneous images of the topography, of the time-averaged tunneling current and of the cantilever excitation, i.e. the damping, at constant
$A$ and $\Delta f$. All images showed characteristic features (adatoms and corner holes) of the dimer-adatom-stacking fault (DAS) model of the $7 \times 7$ reconstruction [11]. Point defects, e.g. missing adatoms appearing at the same locations in all three images; this convincingly proved true atomic resolution. Surprisingly, the contrast in the damping appeared inverted with respect to the other two images. Other issues are whether $\Delta f$ jumps at the onset of covalent bonding between dangling bonds at the tip apex and on adatoms of the sample [10, and whether optimum atomic-scale resolution is obtained at a closer separation [10, in a soft intermittent contact mode [7] or even in the range where $\Delta f$ increases with decreasing tip sample separation [6]. In this contribution we show how relevant information about those issues and quantities measured in large-amplitude DFM can be effectively extracted from classical molecular dynamics simulations despite the enormous discrepancy in time scales between the tip oscillation and atomic motions.

The rest of the paper is organized as follows: the next section describes the model and computational details; results are presented in the following two sections; conclusions are summarized at the end.

\section{THE MODEL}

The Si(111) sample is represented by a slab of eight layers with atoms coupled via the well-known short-range Stillinger-Weber (SW) potential [12] which, besides a pair interaction, contains a three-body term favoring tetrahedral covalent bonding. Periodic boundary conditions are applied laterally to a nearly square supercell encompassing four $7 \times 7$ unit cells. Atoms in the two bottom layers are fixed in bulk-like positions. Atoms in the following three layers are allowed to move, but their average ki- 
netic energy is controlled by a thermostat. The $\mathrm{Si}(111)-$ $7 \times 7$ reconstruction was initiated by removing and shifting atoms in the next two layers, and placing adatoms in the top layer so as to approximately obtain the geometry of the dimer-adatom-stacking fault(DAS) model [11]. Dimers spontaneously formed between faulted and unfaulted half-cells in the third layer and adatoms equilibrated $2.7 \AA$ away from their second layer neighbors shortly after molecular dynamics was started. Subsequent simulations were initiated with this equilibrated structure.

Focusing on short-range covalent interactions, which presumably give rise to atomic-scale contrast in the noncontact regime, we model the tip by a sharp pyramidal silicon cluster with 34 atoms in 6 layers. Like AFM tips etched out of $\mathrm{Si}$, it has a [001] axis. They were kept at bulk-like positions in order to isolate effects due to sample deformation and dynamics. Because the SW potential vanishes beyond $3.8 \AA$, the longer-ranged pair part of a potential [13] which was fit to a first-principles computation of the interaction between Si layers was added when considering the interaction between the tip and sample atoms. This potential has a decay length $\lambda=0.8 \AA$ close to that found in similar recent calculations [14] of the interaction between a $\mathrm{Si}_{10}$ cluster tip and a $\mathrm{Si}(111)$ surface. Compared to Ref.14, the force $F_{z}$ computed for the same $\mathrm{Si}_{10}$ tip above an adatom using our interaction has a similar tail, but reaches a minimum (maximum attraction) at a distance $z=3.0 \AA$ from the unperturbed top layer which is too strong $(-7 \mathrm{nN}$ vs. $-2.25 \mathrm{nN})$. Similar values are obtained with our model tip. In the non-contact range, our results are thus at least semiquantitatively correct.

The coordinates of the all moving atoms are updated according Newton's law, using a $5^{\text {th }}$-order Gear predictor-corrector algorithm 15] with a time step of $0.4 \mathrm{fs}$ as advocated by Stillinger and Weber [12]. The average temperature is controlled by a first-order feedback loop 15]. The thermalization time $\tau_{T}$ can be chosen such that the coupling to the thermostat is weak, but the discrete vibrational spectrum of our finite system is broadened without appreciable distortions. Because the SW potential led to appreciable surface diffusion at room temperature (which is not realistic), the setpoint temperature was fixed at $100 \mathrm{~K}$ to ensure only small oscillations about the $7 \times 7$ equilibrium structure. Another important concern is the discrepancy between practical times for simulations and relevant ones in DFM experiments, e.g. the tip oscillation period $1 / f$ (few ps vs. few ms). Because $\Delta f$ and the distance-dependent contribution to the damping [8] are induced by the tip-sample interaction, the cantilever dynamics per se can be ignored if only its stationary oscillation on resonance is considered.

In order to track tip-induced changes, in each simulation we recorded snapshots of atomic positions, as well as the time evolution of the following quantities: the center-of-mass of all moving atoms, their kinetic energy(temperature), the coordinates of the tip apex and of selected adatoms, all components of forces on the tip and on those adatoms, the work done on the tip and the heat transfer to the thermostat.

\section{QUASISTATIC SCANS}

According to perturbation theory [4, 16], a normalized frequency shift independent of $k$ can be computed in the non-contact range from the z-dependence of $F_{z}$ (including possible reversible deformations),

$$
k A \frac{\Delta f}{f_{0}}=-f_{0} \int_{0}^{\frac{1}{f_{0}}} F_{z}[z(t)] \cos \omega t f_{0} d t
$$

where $\omega=2 \pi f_{0}, z=d+A(1+\cos \omega t), d$ is the minimum tip-surface distance. In the large amplitude limit $A>>d$, constant $\Delta f$ approximately corresponds to constant $F_{z}(d)$ at the turning point $z=d$. Quasistatic force-distance characteristics were obtained by approaching the tip at a constant velocity $v_{z}<<v_{c}=\lambda f_{\max } \simeq$ $1000 \mathrm{~m} / \mathrm{s}, f_{\max }=16 \mathrm{THz}$ being the highest natural frequency of our system (adatom vibration against neighboring atoms). Such approach curves are essentially $v_{z^{-}}$ independent and reversible down to a site-dependent separation $z_{c}$ (e.g. $3.75 \AA$ above adatoms) but strongly $v_{z}$-dependent and hysteretic below because irreversible symmetry-lowering jumps of the nearest adatom(s) are then induced, leading to a transverse force in the $\mathrm{nN}$ range. Although our interaction overemphasizes such instabilities, we expect them to develop below the inflection point in $F_{z}(z)$, even before the net attraction reaches a maximum. Indeed, this inflection signals the appearance of repulsive forces on the nearest adatoms which can be efficiently released by sideways displacement.

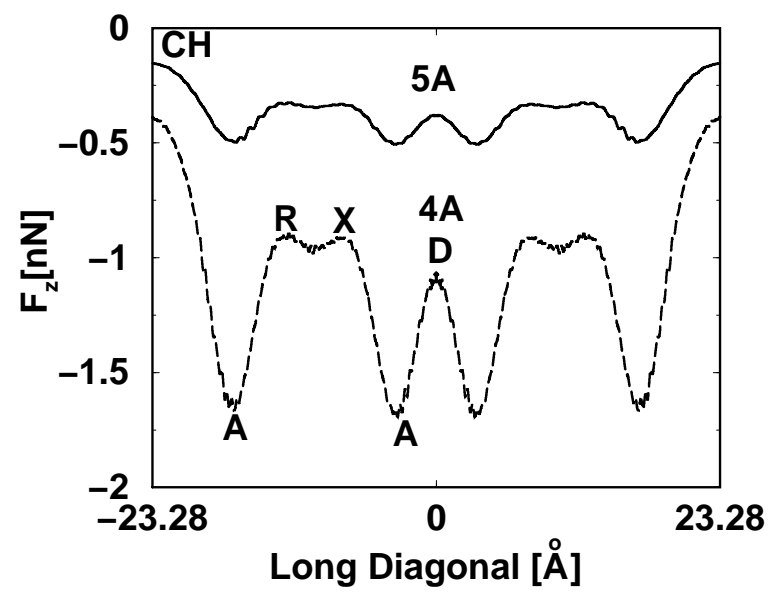

FIG. 1. Variation of the normal force on the tip along the long diagonal of the $\mathrm{Si}(111)-7 \times 7$ unit cell recorded at $2 \AA / p s$ at a height $\mathrm{z}=5$ and $4 \AA$ measured from the unperturbed adatom layer. The symbols $\mathrm{CH}, \mathrm{A}, \mathrm{R}, \mathrm{X}, \mathrm{D}$ denote the positions of the corner hole, corner and center adatoms, rest atom, hollow site and dimer row(short diagonal) of the underlying reconstructed surface 
Below the highest $z_{c}$ constant height scans recorded at constant velocity exhibit friction and wear, adatoms being successively picked up and sometimes redeposited by the moving tip. Above that critical height such scans are reversible and, as shown in Fig. 1, exhibit a contrast with site-specific features in $F_{z}$ which becomes more pronounced at smaller $z$. Whereas maximum attraction above adatoms is expected [5, 14, note that $F_{z}$ is the same above the rest atom $(\mathrm{R})$ and above the hollow site (X) in the adjacent triangle between adatoms, although a recessed dangling bond exists only at the R-site. This implies that attraction to the adatoms dominates the picture. Because our model takes no account of charge transfer effects [14, both cell halves and all adatoms appear equivalent.

Force-distance characteristics recorded at closely spaced points along a particular line can be used to reconstruct scans measurable under all possible feedback operation modes. We checked that the constant height scan at $z=4 \AA$ in Fig. 11 essentially coincides with the corresponding scan reconstructed in this fashion. The same procedure can be used to reconstruct scans at constant normalized frequency shift using Eq.(1) with the time variable eliminated in favor of $\tilde{z}=A \cos \omega t$ in order to take advantage of the dense set of data points recorded in approach simulations,

$$
k A \frac{\Delta f}{f_{0}}=-\frac{1}{\pi A} \int_{-A}^{A} \frac{F_{z}(d+A+\tilde{z}) \tilde{z}}{\sqrt{A^{2}-\tilde{z}^{2}}} d \tilde{z}
$$

The rhs of Eq.(2) is independent of spring constant $k$ and oscillation frequency. From the reconstructed topographical scans along the long diagonal shown in Fig. 2 we see that the constant frequency shift scan is close to the constant force curve. The contrast of the former is slightly reduced due to the non-uniform weight multiplying $F_{z}$ in rhs of Eq.(2). As expected, the overall contrast is inverted with respect to the constant height scans in Fig. 1. Furthermore, the apparent height difference between corner hole and adatom sites is approximately $1.7 \AA$, i.e. larger than in measurements at constant $\Delta f$, except one 10]. This is likely due to our neglect of interactions other than covalent bonding. The contrast we observe is the maximum possible one for a given normalized frequency shift or force; the inclusion of Van der Waals and long range electrostatic interactions would reduce the contrast. The main advantage of constant height scans is that the contrast should be independent of such longrange interactions, because they are mainly determined by many atoms away from the tip apex.

In order to assess the resolution of native surface defects, we first created representative ones by (i) removing a center adatom and (ii) placing an additional one at one of lowest energy surface sites $H_{3}$ [17 which is next to a corner adatom, and then relaxed the sample. Afterwards we recorded the constant height scans plotted in Fig. 3 together with the scan above the perfect surface already shown in Fig. 1. Compared to the native adatom sites, we observe an almost twice as strong attraction above the dimer formed between the additional adatom and the nearby corner adatom, but it is not possible to differentiate the two partners. On the other hand, the missing adatom produces a maximum almost as high and slightly broader than the corner hole. This appearance is consistent with that of deep minima associated with a missing adatom in constant $\Delta f$ images [8].

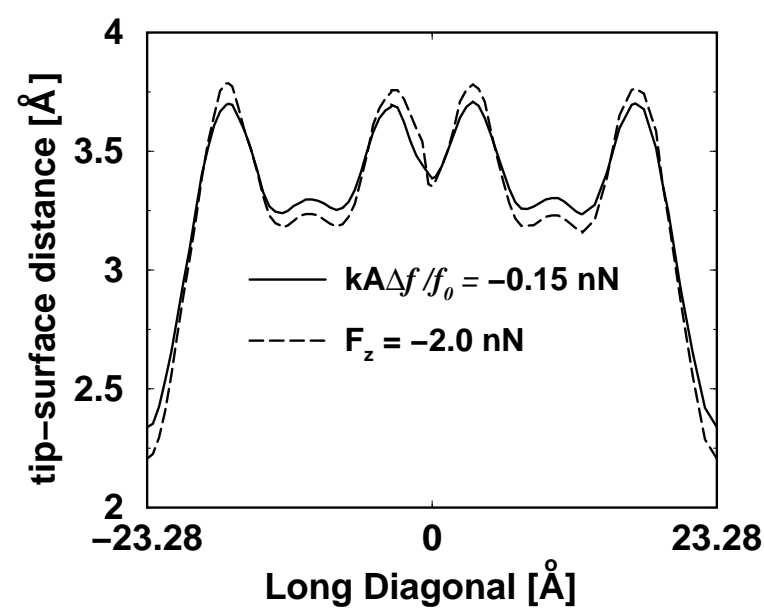

FIG. 2. Topographical scans at constant force (dashed curve) and constant normalized frequency shift (full curve) recorded under the same conditions as Fig. 1. Both curves were computed by interpolation from quasistatic force-distance characteristics.

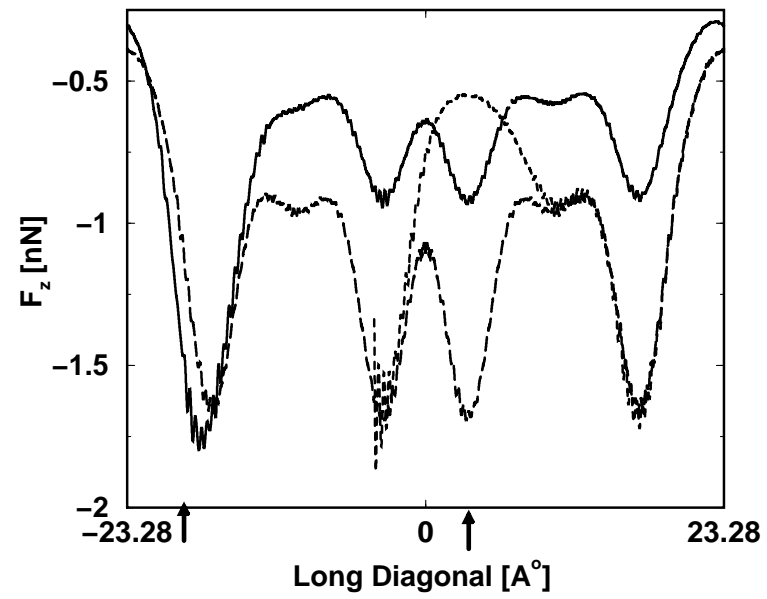

FIG. 3. Constant height scans recorded under the same conditions as Fig. 1 in the presence of a missing center adatom (short-dashed curve, $\mathrm{z}=4 \AA$ ), an additional adatom bound to a corner adatom (full curve, $\mathrm{z}=4.5 \AA$ ) compared a similar scan above the perfect surface (dashed curve, $\mathrm{z}=4 \AA$ ). The position of the missing (additional) adatom is indicated by the right (left) arrow on the horizontal axis. The oscillations appearing at the minima above adatoms are induced by the scan onset or by thermal fluctuations. 


\section{PERIODIC TIP OSCILLATION}

In order to determine the distance-dependent damping attributed to the excitation of phonons [8], we have performed dynamical simulations in which the tip was sinusoidally driven so that it comes within a few angstroms of the sample. As expected, the time-dependent force on the tip consist of a periodic series of narrow pulses of width $\sqrt{\lambda / A} / f$ slightly modulated by thermal fluctuations. Fig. 4 illustrates an extreme case where this minimum distance is smaller than $z_{c}$, so that the center adatom under the tip would be destabilized in a quasistatic scan. In view of the(unrealistically) narrow pulse width, a jump to an energetically more favored position on the side of the original site occurs only after the ninth pulse. Under typical experimental conditions the pulse width would be $\approx 1 \mu s$ i.e. encompassing more than one million adatom vibrations, a quasistatic situation. Nevertheless, a small amount of energy can be transferred to acoustic phonons, either directly or via anharmonic decay of local vibrations excited by the force pulses. In our simulations interaction-induced damping due to both processess [8] can be obtained if the inverse interaction time $f \sqrt{A / \lambda}$ lies between $f_{\max }$ and the frequency of the lowest mechanical resonance of our slab (about $1 \mathrm{THz}$ ). Thus that the imposed frequency cannot be much below that lower limit.

To visualize energy transfer we calculate the work done by the tip as $W=-\int F_{z} v_{z} d t$. From Fig. 4 we see that apart from quasi-reversible spikes accompanying the force pulses, $W$ exhibits an irreversible increase, which comes about because sample atoms do not quite respond adiabatically. This results in a slight asymmetry of the force pulses. This irreversible part of the work goes into vibrational energy which is then shared between different modes i.e. turns into heat. This transformation is somewhat assisted by our weakly coupled feedback loop which regulates the total kinetic energy of all thermalized atoms according to the equipartition rule.

The thermostat time constant $\tau_{T}$ can be chosen shorter than the period so that equilibration is practically achieved between successive pulses. The heat transferred to the thermostat then looks like a staircase as a function of time, and the average transfer rate coincides with the mean power transferred by the tip (overall energy conservation) as demonstrated elsewhere 18. In those earlier simulations, we observed that for a given force at the minimum distance $d$ the energy transfer rate was stronger at the $\mathrm{CH}$ site than at the $\mathrm{X}$ site, in apparent agreement with the experimentally observed inverted contrast in the damping. As expected the damping contrast was in accordance with the topography if the tip was driven with the same amplitude, but coming within the same minimum distance $\mathrm{d}=3.4 \AA$. The observation of a contrast in the damping [8] at a constant force $F_{z}(d)$ is a delicate issue because such a contrast would vanish if the substrate were treated as a continuum subject to delta function like pulses 19. In recent dynamic simulations we managed to detect a damping roughly proportional to $F_{z}^{2}(d)$ in the non-contact range, as expected for a linear response. We found that it is maximum above adatoms, i.e. at sites where the distribution of $F_{z}$ among nearby atoms is most localized laterally.
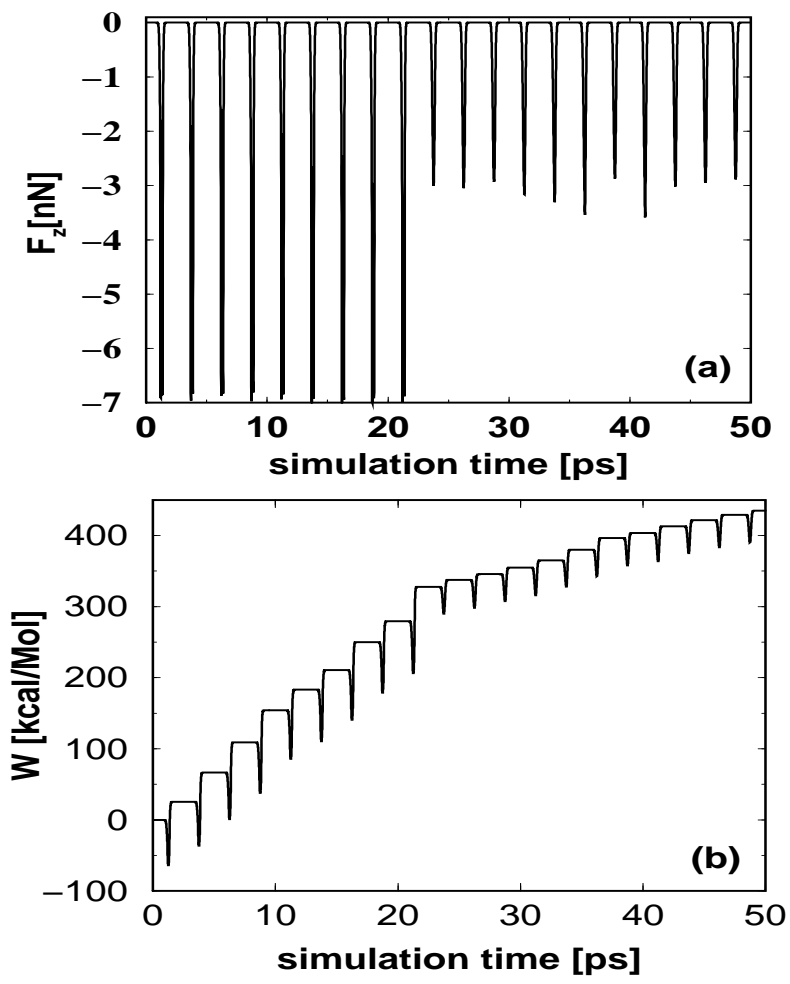

FIG. 4. Top: Time-dependent normal force on the tip oscillated at $0.4 \mathrm{Thz}$ with an amplitude $\mathrm{A}=20 \AA$ above a center adatom to a minimum distance $3.15 \AA$ from the unperturbed adatom layer. Bottom: Corresponding evolution of the work on the tip; the energy transferred to the sample after each pulse is converted in to heat(see text).

\section{CONCLUSIONS AND ACKNOWLEDGMENTS}

Molecular dynamics simulations can be conducted and analyzed effectively to provide valuable information unavailable in experiments, as well as to compute measurable properties (resonance frequency shift and damping) and corresponding scan lines. This has been illustrated for the $\operatorname{Si}(111)-7 \times 7$ surface. Bearing in mind that our model tip is extremely sharp and that long-range interactions are ignored, the agreement with experimental constant $\Delta f$ scans is quite satisfactory. As with any simulations, some details are sensitive to the underlying model. The interactions we assumed tend to unduly favor adatom jumps along the surface and to the tip below a separation $z_{c}$ which appears somewhat large. Nevertheless, such a critical distance is expected to appear before the net force reaches a minimum (maximum attraction) 
upon approach. It is therefore still difficult to understand how stable DFM images at constant $\Delta f<0$ can be obtained following a jump in $\Delta f$ [10] or in the range where $\Delta f$ increases with decreasing tip-sample separation [6]. The inverted contrast sometimes observed in the topography [6] and in the damping [8] also remains unexplained. New attempts to reproduce such measurements, with careful attention to possible feedback artifacts and to the relevant range of setpoints are therefore very desirable. More work is also needed to understand the detailed dissipation pathways and to extrapolate the computed damping for comparison with measured values.

The authors thank Prof. H.-J. Güntherodt and the Swiss National Research program NFP36 "Nanosciences" for encouragement and support, T. Bonner for initiating this simulation and his molecular dynamics code, and M. Bammerlin, R. Bennewitz, M. Guggisberg and R. Lüthi for helpful discussions.

* Corresponding author, email: abduxukur.abdurixit@unibas.ch, fax: +41-61-267 37 84/95, tel: +41-61-267 3761

[1] F.J. Giessibl, Science 267 (1995) 68

[2] S. Kitamura and H. Iwatsuki, Jpn. J. Appl. Phys. 34 (1995) L145

[3] T.R. Albrecht, P. Grütter, D. Horne and D. Rugar, J. App. Phys. 69 (1991) 668

[4] F.J. Giessibl, Phys. Rev. B, 56 (1997) 16010

[5] R. Lüthi, E. Meyer, M. Bammerlin, A. Baratoff, T. Lehmann, L. Howald, Ch. Gerber, and H.-J. Güntherodt, Z. Phys. B 100 (1996) 165

[6] P. Güthner, J. Vac. Sci. Technol. B 14 (1996) 2428

[7] R. Erlandsson, L. Olsson and P. Martensson Phys Rev. B 54 (1996) 8309

[8] R. Lüthi, E. Meyer, M. Bammerlin, A. Baratoff, L. Howald, Ch. Gerber, and H.-J. Güntherodt, Surf. Rev. Lett. 4 (1997) 1025. The image in Fig. 3(b) of that conference report should represent the topography at a constant frequency shift, it was unfortunately inverted and mislabelled.

[9] N. Nakagiri, M. Suzuki, K. Obiguchi and M. Sugimura, Surf. Sci. 373 (1997) L329

[10] T. Uchihashi, Y. Sugawara, T. Tsukomoto, M. Ohta, S. Morita, M. Suzuki, Phys Rev. B 56 (1997) 9834

[11] K. Takayanagi, Y. Tanishiro, M. Takahashi, and S. Takahashi, J. Vac. Sci. Tech. A 3 (1985) 1502

[12] F. H. Stillinger and T. A. Weber, Phys. Rev. B 31 (1985) 5262

[13] R. Biswas and D. R. Hamann, Phys. Rev. Lett. 55 (1985) 5262

[14] R. Perez, I. Stich, M.C. Payne and K. Terakura, Phys. Rev. B 58 (1998) 10835

[15] M. P. Allen and D. J. Tildesley, Computer Simulation of Liquids (Oxford University Press, 1989)
[16] A. Baratoff and A. Abdurixit, presented at NC-AFM 98 and in preparation

[17] K. Cho and E. Kaxiras, Europhy. Lett. 39 (1997) 287

[18] A. Abdurixit, T. Bonner, A. Baratoff and E. Meyer, presented at STM'99 and NC-AFM 99.

[19] U. Dürig, Surf. Interface Anal. 27 (1999) 467 